\title{
OCCUPATIONAL CHEMICAL EXPOSURES AND
} MENINGIOMA

Laura MacCalman, ${ }^{1}$ Martie van Tongeren, ${ }^{1}$ Geza Benke, ${ }^{2}$ Sarah Fleming, ${ }^{3}$ David McLean, ${ }^{4}$ Martine Hours, ${ }^{5}$ Daniel Krewski, ${ }^{6}$ Marie-Elise Parent, ${ }^{7}$ Siegal Sadetzki, ${ }^{8}$ Brigitte Schlehofer, ${ }^{9}$ Jack Siemiatycki, ${ }^{10}$ Elisabeth Cardis, ${ }^{11}$ Laurel Kincl, ${ }^{11}$ Lesley Richardson ${ }^{10} 10 \mathrm{M}$, Edinburgh, UK; ${ }^{2}$ Monash University, Melbourne, Australia; ${ }^{3}$ University of Leeds, Leeds, UK; ${ }^{4}$ Massey University, Wellington, New Zealand; ${ }^{5}$ Université Claude Bernard, Lyon, France; ${ }^{6}$ Univeristy of Ottawa, Ottawa, Canada; ${ }^{7}$ University of Quebec, Quebec, Canada; ${ }^{8}$ Gertner Institute, Tel Aviv, Israel; ${ }^{9}$ DFKZ, Heidelberg, Germany; ${ }^{10} \mathrm{CRCHUM}$, Montreal, Canada; ${ }^{11}$ CREAL, Barcelona, Spain

10.1136/oemed-2011-100382.199

Objectives Currently little is known about risk factors associated with meningioma with the exception of ionising radiation exposure, although there are many suggestions in the literature. As meningioma affects proportionately more females, hormones may play a role. A case control study was undertaken to examine possible associations between specific brain cancers and occupational exposures. We present the analysis of risk of meningioma associated with occupational chemical exposures.

Methods The Finnish Job Exposure Matrix (FinJEM) was modified and applied to lifetime work histories of the subjects to obtain occupational exposures to each of 29 agents. Exposures were calculated up to 5 years before the reference date to allow for latency. Case-control analysis examined whether exposure to each agent affected the risk of meningioma, using unconditional logistic regression adjusting for age, sex, country and education. A variety of exposure metrics were investigated including cumulative exposure, ever/never and quintiles of exposure level.

Results Information was available on 1925 cases of meningioma and 5813 controls from 7 countries. A high proportion of subjects were determined to be occupationally exposed to PAH and benzo[a]pyrene ( $80 \%$ ) but no more than $20 \%$ of subjects were exposed to the other agents considered. A slight increase in risk of meningioma was found with higher occupational exposures to chromium and nickel (OR=1.2-1.7).

Conclusions Exposure to a small number of agents resulted in an elevated risk of meningioma; these suggested links need more detailed examination. Further analyses will include examination of subgroups and of time windows of exposure. 